\title{
Highly Selective and Efficient Synthesis of 7-Aminoquinolines and Their Applications as Golgi-Localized Probes
}

\author{
Jiahui Chen, ${ }^{\dagger}$ Huijing Liu, ${ }^{\dagger}$ Li Yang, ${ }^{\ddagger}$ Jun Jiang, ${ }^{\ddagger \odot}$ Guoqiang Bi, ${ }^{\dagger}$ Guoqing Zhang, ${ }^{\ddagger \odot}$ Guisheng Li, ${ }^{*}, \S_{\odot}$ \\ and Xiaofeng Chen*, ${ }^{*}$ \\ ${ }^{\S}$ School of Environmental and Geographical Sciences, Shanghai Normal University, Shanghai 200234, China \\ ${ }^{\dagger}$ School of Life Sciences, University of Science and Technology of China, Hefei 230026, China \\ ${ }^{*}$ Hefei National Laboratory for Physical Sciences at the Microscale, University of Science and Technology of China, Hefei 230026, \\ China
}

Supporting Information

ABSTRACT: Quinoline derivatives have extensively been used for both pharmaceutical agents and bioimaging. However, typical synthesis of quinoline derivatives is generally through strong acid/base-catalyzed or metal-catalyzed methods at high temperatures. Here we report a catalyst-free synthesis of 2,4-disubstituted 7 -aminoquinolines with high selectivity and good yields via the introduction of a trifluoromethyl group. It is discovered that quinolines containing both amino and trifluoromethyl groups exhibit
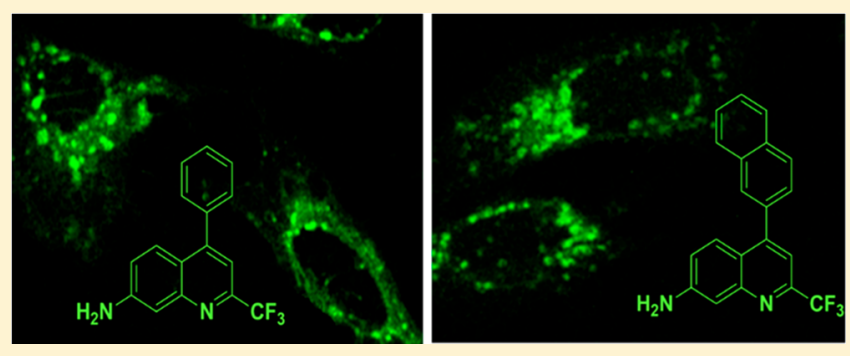
strong intramolecular charge-transfer fluorescence with large Stokes shifts. We further applied the obtained quinolines to live-cell imaging and found that some of the derivatives can target specifically Golgi apparatus in various cell lines (HeLa, U2OS, and 4T1 cells) in vitro and the colocalization with commercial Golgi marker is retained during the mitosis in HeLa cells. Moreover, the quinoline dyes can also be used for Golgi apparatus imaging with two-photon fluorescence microscopy. These results provide new insights into developing low cost Golgi-localized probes.

KEYWORDS: Quinoline, selective synthesis, intramolecular change transfer, fluorescence, cell imaging

uinolines and their derivatives play a crucial role in organic chemistry due to the applications in pharmaceuticals as well as advanced functional materials. ${ }^{1-4}$ As the important "star molecules" in various research areas, quinolines continued to receive extensive research interests in the past few years, particularly as the core scaffold to construct medicine molecules $^{5-8}$ and fluorescent probes for sensing. ${ }^{9-13}$ Given the importance of the quinoline scaffold in the fields of both pharmaceutical and organic chemistry, synthesis of quinoline derivatives has received tremendous attention since Skraup first reported the classical synthetic method of quinoline in $1880 .{ }^{14}$ Over the past few decades, numerous methods based on various mechanisms, including Conrad-Limpach-Knorr, ${ }^{15}$ Friedländer, ${ }^{16}$ Doebner-von Miller, ${ }^{17}$ Pfitzinger, ${ }^{18}$ or Combes, ${ }^{19}$ have been developed for the preparation of substituted quinolones. However, multiple synthetic steps and harsh reaction conditions, such as high temperatures, strong base, acid or metal catalysts, still limit the applications of these strategies. Therefore, direct catalyst-free approaches with good yields remain highly valuable to broaden the application of quinoline derivatives.

As one of the common cellular organelles, Golgi apparatus is the major collecting, processing, and dispatching station of proteins to be modified and delivered for secretion. ${ }^{20}$ Since involved in such vital intracellular activities, disruption of Golgi apparatus functions could cause many organ lesions such as eye, kidney, and liver diseases. ${ }^{21}$ Thus, it is of great significance to develop specialized probes to mark Golgi apparatus. Considering most commercial Golgi markers generally are difficult to be synthesized with complicated structures or high cost, ${ }^{22-24}$ the development of Golgi-localized small molecule probes has still remained challenging. The application of quinoline derivatives as cell organelle probes is rarely explored, even though it has been widely applied to construct medicine molecules and analyte probes. Moreover, quinoline primarily fluoresces in the near UV region, it is desirable to shift its absorption and emission signals to longer wavelengths without significantly extending the molecular size for biosensing and bioimaging. In this context, charge-transfer state could be used as an effective strategy to mediate excited states. ${ }^{25,26}$ As a strongly electron-withdrawing group, trifluoromethyl group has been used to form charge-transfer states with electron-donating groups. ${ }^{27-29}$ Meanwhile, biological and medicinal studies have

Received: March 19, 2019

Accepted: May 28, 2019

Published: May 28, 2019 
demonstrated that fluorine-containing compounds also exhibit enhanced biological properties. ${ }^{30-32}$

Considering both feasible synthetic routines and biological applications of quinolines, we propose here to conduct a catalyst-free reaction of $m$-phenylenediamine with unsymmetric 1,3-diketones containing a trifluoromethyl group to synthesize various substituted 7-aminoquinolines as novel fluorophors in good yields. Introduction of such a trifluoromethyl group avoids the use of concentrated acid to promote the condensation as well as the subsequent treatment with excess amount of base to liberate the quinoline as previously reported. ${ }^{33}$ And the strongly electron-withdrawing trifluoromethyl group also potentially enhances intramolecular change transfer (ICT) state of the 7-aminoquolines between the strongly electron-donating amine group and the trifluoromethyl group, thus shifting the absorption and emission of the compounds to the longer wavelengths in polar media.

We first tested the reaction of $m$-phenylenediamine with 4,4,4-trifluoro-1-phenylbutane-1,3-dione. As shown in Scheme 1 , after a chloroform solution of the two compounds were

\section{Scheme 1. Syntheses of 7-Aminoquinolines}

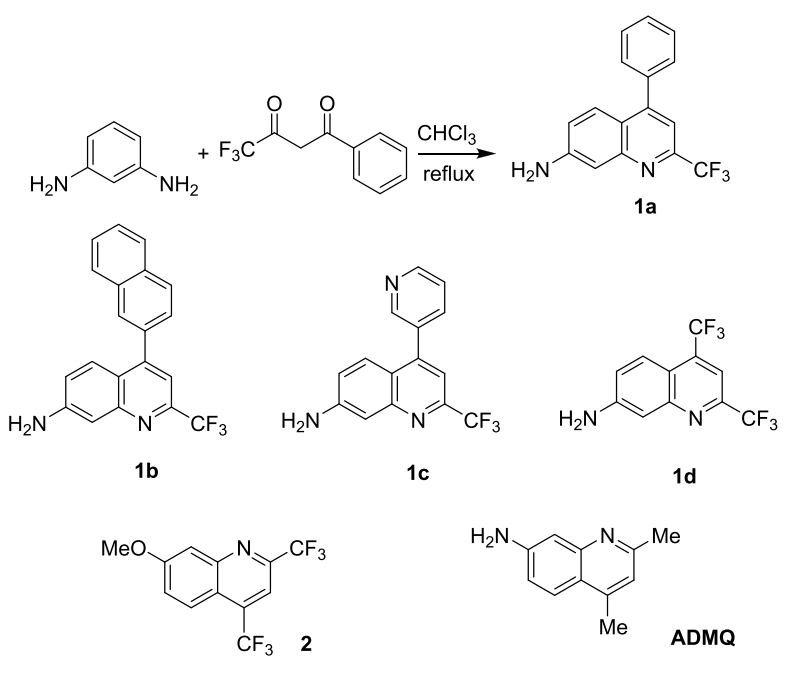

heated at reflux for $\sim 10 \mathrm{~h}$, rotary evaporation of the solvent directly led to light green needle crystals of the product in $88 \%$ yield. No strong acid or other catalyst was needed to promote this reaction. Surprisingly, this reaction was found to be able to proceed in a solvent-free solid state! The two starting reactants were thoroughly ground in a mortar, and the resulted pale mixture turned light green overnight at room temperature in the air. ${ }^{1} \mathrm{HNMR}$ spectroscopic analyses confirm the only product structure of $\mathbf{1 a}$, avoiding the formation of regioisomeric products that one would expect for the reaction of $\mathrm{m}$ phenylenediamine with unsymmetric 1,3-diketones. Here, we propose the reaction routine in Scheme 2: the first step is the nucleophilic addition (NA) between one amine group of $m$ phenylenediamine and the ketone group adjacent to trifluoromethyl group, due to its strong electronegativity. Then a second condensation of the other ketone group with the aromatic ring at 6-position, not 2-position, follows, contributing to the octet rule, to form the final single product 1a. Therefore, using such trifluoromethyl substituted 1,3diketones has directed a highly selective and efficient condensation to form the $4-\mathrm{CF}_{3}$-substituted 7 -aminoquinoline as a single product.
Scheme 2. Proposed Formation Mechanism of 7Aminoquinolines

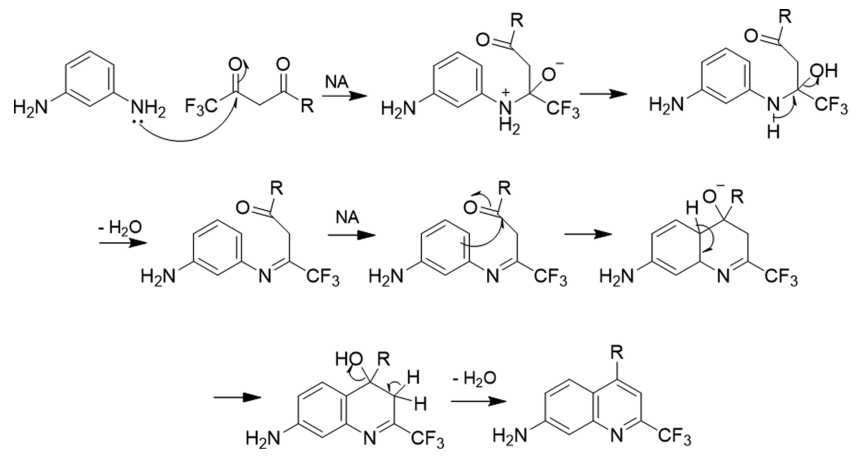

In a similar way, we also conducted the reaction with various substituents on the unsymmetric trifluoromethylated 1,3diketones to test the versatility of the method to introduce a trifluoromethyl group. Compounds $\mathbf{1 b}-\mathbf{1 d}$ were successfully obtained from the corresponding unsymmetric 1,3-diketones in good yields, demonstrating the strategy could be widely applied. Moreover, when $m$-phenylenediamine was reacted with hexafluoroacetylacetone, the reaction condition can be so mild that the product $1 \mathbf{d}$ could even be produced in water at room temperature (Figure S1), which indicates that the two trifluoromethyl groups further accelerate the reaction.

We then studied the fluorescence ICT properties of the 2,4disubstituted 7 -aminoquinolines in various solvents. For comparison, we prepared 7-methoxyquinoline $\mathbf{2}$ with a methoxy group that is less electron-donating than the amine group and 7-amino-2,4-dimethylquinoline ADMQ with two methyl groups that are less electron-withdrawing than the trifluoromethyl group, and compared their optical properties with the new quinolines. A summary of the optical characterizations of compounds $\mathbf{1 a}-\mathbf{d}, \mathbf{2}$, and ADMQ is presented in Table 1. The absorption and emission spectra are also provided (Figures 1 and S2-S7), along with photos showing the solvatochromic fluorescent emissions (Figures S8-S13). In general, the trifluoromethyl-substituted 7-aminoquinolines (1a-1d) show rather strong absorption in the near UV to blue light region. Absorption maxima exhibit gradual bathochromic shifts as the solvent polarity increases, e.g., $\lambda_{\max }=$ 365-368 $\mathrm{nm}$ in $n$-hexane and $\lambda_{\max }=389-399 \mathrm{~nm}$ in methanol. The visual emission color changes from violet in $n$-hexane $\left(\lambda_{\text {em }}=407-435 \mathrm{~nm}\right)$ to greenish yellow $\left(\lambda_{\text {em }}=507-\right.$ $537 \mathrm{~nm})$ in $\mathrm{MeOH}$, indicating increased excited-state dipole moments of these molecules. Presumably this is due to an ICT process from the $-\mathrm{NH}_{2}$ lone pair to the vicinity of the electron-withdrawing $-\mathrm{CF}_{3}$ group. As a result, when $-\mathrm{NH}_{2}$ is replaced with a less powerful electron-donating $-\mathrm{OCH}_{3}$ group, or the strong electron-withdrawing group $-\mathrm{CF}_{3}$ with an electron-donating $-\mathrm{CH}_{3}$ group, the quinoline compounds 2 and ADMQ emit in the violet region in all the solvents $(<420$ $\mathrm{nm})$, suggesting that the lowest transition consists of more $\pi-\pi^{*}$. Time-correlated fluorescence lifetimes were also measured in different solutions (Table 1). With increasing solvent polarity, fluorescence lifetimes of the 7-aminoquinolines first lengthen (e.g., 1d: 4.96 to $20.0 \mathrm{~ns}$ from $n$-hexane to ethyl acetate) in moderately polar solvents but start to shorten in strongly polar solvents (e.g., 1d: 7.49 ns in methanol). The trend is typical of ICT compounds discussed in a previous publication. ${ }^{34}$ Briefly speaking, the initially lengthened lifetimes 
Table 1. Optical Properties of Quinoline Compounds 1a-1d, 2, and ADMQ in Different Solutions

\begin{tabular}{|c|c|c|c|c|c|c|c|c|}
\hline solvent $^{a}$ & A & B & $\mathrm{C}$ & $\mathrm{D}$ & $\mathrm{E}$ & $\mathrm{F}$ & G & $\mathrm{H}$ \\
\hline$\varepsilon^{b}$ & 1.88 & 2.38 & 4.81 & 6.02 & 7.58 & 8.93 & 20.56 & 32.66 \\
\hline 1a $\lambda_{\max }(\mathrm{nm})^{c}$ & 368 & 375 & 371 & 384 & 389 & 373 & 387 & 389 \\
\hline$\lambda_{\mathrm{em}}(\mathrm{nm})^{d}$ & 407 & 442 & 468 & 472 & 473 & 449 & 483 & 507 \\
\hline$\tau_{\mathrm{F}}(\mathrm{ns})^{e}$ & 2.89 & 4.56 & 5.82 & 7.22 & 8.04 & 3.63 & 8.93 & 7.84 \\
\hline $\mathbf{1 b} \lambda_{\max }(\mathrm{nm})$ & 367 & 375 & 372 & 387 & 391 & 374 & 389 & 392 \\
\hline$\lambda_{\mathrm{em}}(\mathrm{nm})$ & 409 & 452 & 469 & 477 & 480 & 446 & 487 & 513 \\
\hline$\tau_{\mathrm{F}}(\mathrm{ns})$ & 3.89 & 5.17 & 4.55 & 6.13 & 6.66 & 4.30 & 7.01 & 6.72 \\
\hline 1c $\lambda_{\max }(\mathrm{nm})$ & 365 & 374 & 372 & 382 & 387 & 373 & 386 & 391 \\
\hline$\lambda_{\mathrm{em}}(\mathrm{nm})$ & 435 & 475 & 500 & 503 & 512 & 475 & 517 & 537 \\
\hline$\tau_{\mathrm{F}}(\mathrm{ns})$ & 4.24 & 12.9 & 13.7 & 15.6 & 15.9 & 12.2 & 14.7 & 5.50 \\
\hline $\mathbf{1 d} \lambda_{\max }(\mathrm{nm})$ & 368 & 383 & 379 & 393 & 397 & 380 & 396 & 399 \\
\hline$\lambda_{\mathrm{em}}(\mathrm{nm})$ & 413 & 461 & 484 & 489 & 495 & 464 & 501 & 527 \\
\hline$\tau_{\mathrm{F}}(\mathrm{ns})$ & 4.96 & 15.7 & 17.5 & 19.5 & 20.0 & 16.7 & 19.7 & 7.49 \\
\hline $2 \lambda_{\max }(\mathrm{nm})$ & 336 & 341 & 341 & 341 & 342 & 341 & 342 & 341 \\
\hline$\lambda_{\mathrm{em}}(\mathrm{nm})$ & 376 & 398 & 392 & 402 & 402 & 396 & 406 & 417 \\
\hline$\tau_{\mathrm{F}}(\mathrm{ns})$ & 0.68 & 3.97 & 2.25 & 4.18 & 4.16 & 2.57 & 5.19 & 7.58 \\
\hline ADMQ $\lambda_{\max }$ & 339 & 344 & 350 & 347 & 344 & 343 & 349 & 351 \\
\hline$\lambda_{\mathrm{em}}(\mathrm{nm})$ & 374 & 393 & 407 & 401 & 398 & 390 & 408 & 429 \\
\hline$\tau_{\mathrm{F}}(\mathrm{ns})$ & 0.02 & 3.73 & 6.08 & 4.55 & 2.10 & 2.88 & 5.28 & 0.17 \\
\hline
\end{tabular}

${ }^{a} \mathrm{~A}, n$-hexane; B, toluene; C, chloroform; D, ethyl acetate; E, tetrahydrofuran; F, dichloromethane; G, acetone; H, MeOH. ${ }^{b}$ Dielectric constant of the solvent. ${ }^{c} \lambda_{\max }=$ absorption maximum in solution. ${ }^{d} \lambda_{\mathrm{em}}=$ emission maximum in solution (excited at 365 nm). ${ }^{e} \tau_{\mathrm{F}}=$ pre-exponent-weighted fluorescence lifetime.

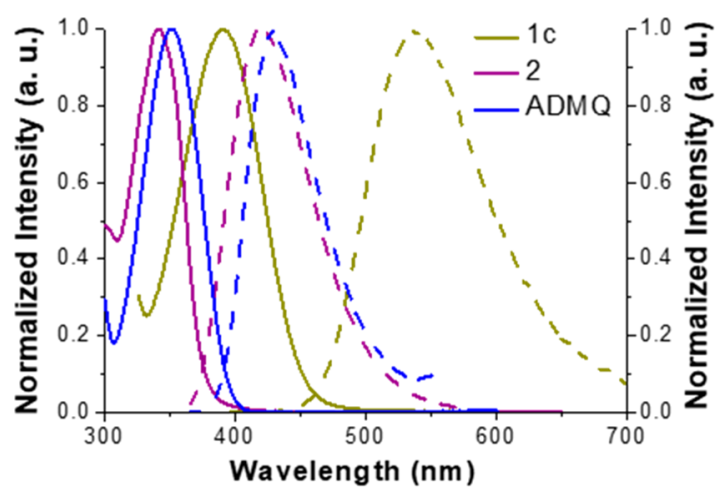

Figure 1. Normalized absorption (solid line) and emission spectra (dashed line) of 1c, 2, and ADMQ in methanol solutions.

are due to a more forbidden ICT character in moderately polar solvents compared to a pure $\pi-\pi^{*}$ transition because the origin and destination orbitals do not entirely overlap in space. Meanwhile, when ICT becomes more dominating in very polar solvents, more dramatic electron redistribution between the donor and acceptor moieties in the excited state can result in subsequent large nuclear reorganization. ${ }^{35}$ The apparent emission lifetime can thus be compromised with increased thermal quenching.

In order to gain further understanding on the optical properties of these compounds, quantum chemistry calculations were performed with the Gaussian09 package. The optimized geometry and electronic structures were simulated with density function theory (DFT) at the B3LYP/6-31G level (Figure 2). The frontier orbitals of compounds $1 \mathbf{a}-\mathbf{1 d}$ possess close energy levels, while the highest occupied molecular orbital (HOMO) of $\mathbf{2}$ is much lower in energy. The higher HOMOs for $1 \mathrm{a}-\mathbf{1 d}$ are evidently caused by the presence of the amino group since the starting material $m$-phenylenediamine (1) has a comparable HOMO energy level. As shown in Figure 2, for 7-aminoquinolines $\mathbf{1 a - 1 d}$, the HOMOs and the

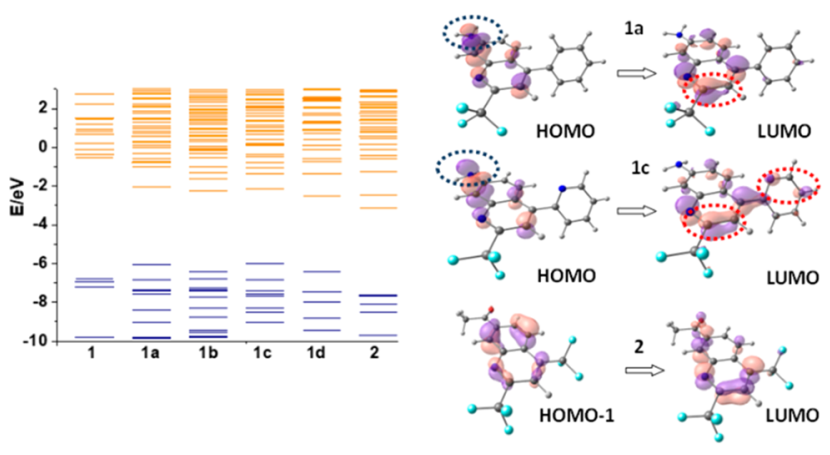

Figure 2. Calculated main origin and destination orbitals involved in the lowest singlet transition states of compounds 1 (m-phenylenediamine), 1a, 1c, and 2. Blue and red circles represent regions with the highest electron density in the ground and in the excited states, respectively.

lowest unoccupied molecular orbitals (LUMOs) are more concentrated on the amino group and near the $-\mathrm{CF}_{3}$ group, respectively, indicating a strong ICT character. For 2, however, the overlap of the HOMO and the LUMO is significantly better and is thus more likely to exhibit a vertical $\pi-\pi^{*}$ state as the lowest transition. Therefore, both the trifluoromethyl group and amino group are essential to form valid ICT states among the new quinolones.

On account of the extensive applications of quinolines in bioimaging and the substantial ICT character of these 7aminoquinolines, we then explored their feasibilities as fluorescent cell imaging agents. All the ICTQs (intramolecular charge transfer quinolines $\mathbf{1 a - 1 d}, \mathbf{2} \mu \mathrm{g} / \mathrm{mL}$ ) in buffer solutions were added to U2OS cells growing at $37{ }^{\circ} \mathrm{C}$ in a $5 \% \mathrm{CO}_{2}$ incubator. We found that $1 \mathbf{a}$ and $\mathbf{1 b}$ exhibited the best brightness and photostability. After $30 \mathrm{~min}$, the live cells were taken under a fluorescence microscope for one-photon imaging. Surprisingly, the compounds tended to specifically locate in the peri-nuclear region of the cells, which might be 
the Golgi apparatus after being internalized (Figures 3a and S14a). In order to exclude the specialty of one kind of cells, we

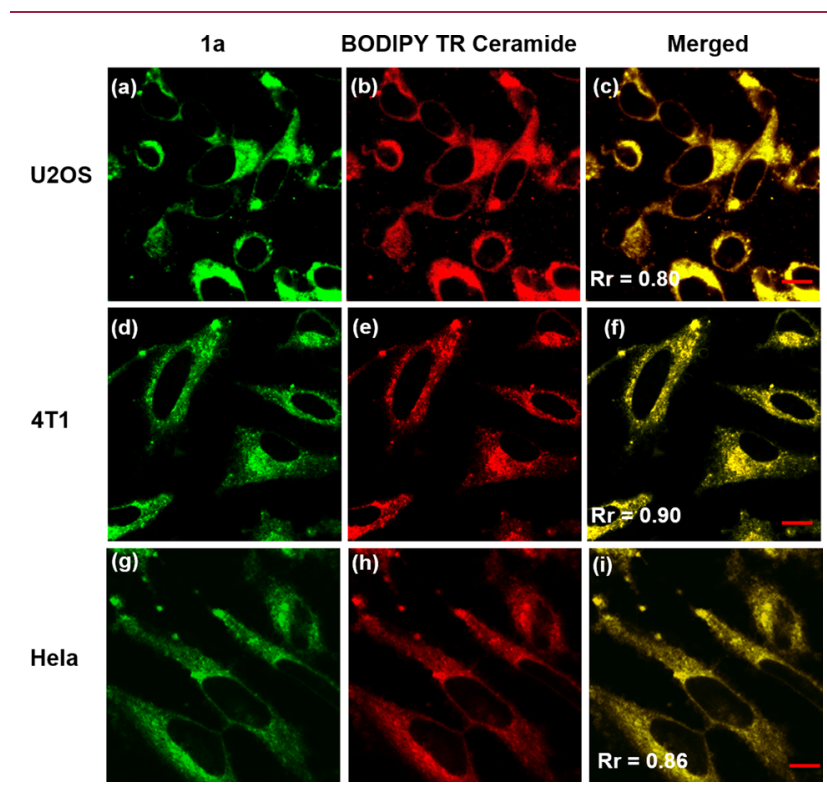

Figure 3. Confocal fluorescence micrographs showing stained Golgi apparatuses by $1 \mathrm{a}\left(\mathrm{a}, \mathrm{d}, \mathrm{g}: \lambda_{\mathrm{ex}}=488 \mathrm{~nm}\right.$; emission range $489-580$ $\mathrm{nm}$ ) and BODIPY TR Ceramide (b, e, h: $\lambda_{\mathrm{ex}}=633 \mathrm{~nm}$; emission range $634-740 \mathrm{~nm}$ ), and the merged images (c, f, i). Scale bar: 10 $\mu \mathrm{m}$.

also conducted the experiments with two other cell lines (HeLa and 4T1 cells) under the same conditions. As shown in Figure 3d, g, 1a and 1b (Figure S14d,g) present similar locations in the Golgi apparatus with strong green emission, which demonstrates that our quinolone dyes possess versatile cell imaging properties. $\mathrm{IC}_{50}$ tests were also conducted to evaluate the toxicity of $\mathbf{1 a}$ and $\mathbf{1 b}$ (Figure S15). The calculated $\mathrm{IC}_{50}$ values for $\mathbf{1 a}$ and $\mathbf{1 b}$ are 3.37 and 3.44 , respectively; thus, the half maximal inhibitory concentrations are 2.34 and 2.72 $\mu \mathrm{g} / \mathrm{mL}$, respectively. Since the experimental concentration of $\mathbf{1 a}$ and $\mathbf{1 b}$ is $2 \mu \mathrm{g} / \mathrm{mL}$ in the present work, no obvious cytotoxicity for these two dyes was noted during all of the cell culture experiments.

To investigate the cellular location specificity of $\mathbf{1 a}$ and $\mathbf{1 b}$, the cells were cross-stained with BODIPY TR Ceramide, a commercial red-emissive marker for the Golgi apparatus, and examined under a Zeiss LSM 710 confocal microscope. All the images for the three cell lines exhibit a complete distribution overlap with the Pearson's correlation coefficients, Rr larger than 0.50 (Figure 3c, f, i and Figure S14c, f, i). Those high Rr values demonstrate that $\mathbf{1 a}$ and $\mathbf{1} \mathbf{b}$ exhibit excellent specificity for Golgi apparatus. ${ }^{36}$ An antigiantin antibody of Golgi apparatus was also cross-stained with $\mathbf{1 a}$ and $\mathbf{1 b}$ to test their specificity for Golgi apparatus in HeLa cells (Figure S16). The $\mathrm{Rr}$ values are 0.65 and 0.83 for $\mathbf{1 a}$ and $\mathbf{1 b}$, respectively, confirming their highly target-specific Golgi apparatus properties. Further, we also examined the colocalization of our dye 1a and BODIPY TR Ceramide during the mitosis in living HeLa cells. The imaging results of different subphases are presented in Figure 4. The $\mathrm{Rr}$ values for prophase, prophase, anaphase, and telophase were found to be $0.77,0.85,0.63$, and 0.86 , respectively, showing that 1a and BODIPY TR Ceramide still show good distribution overlap in different subphases of mitosis. We preliminarily ascribe the highly target-specific

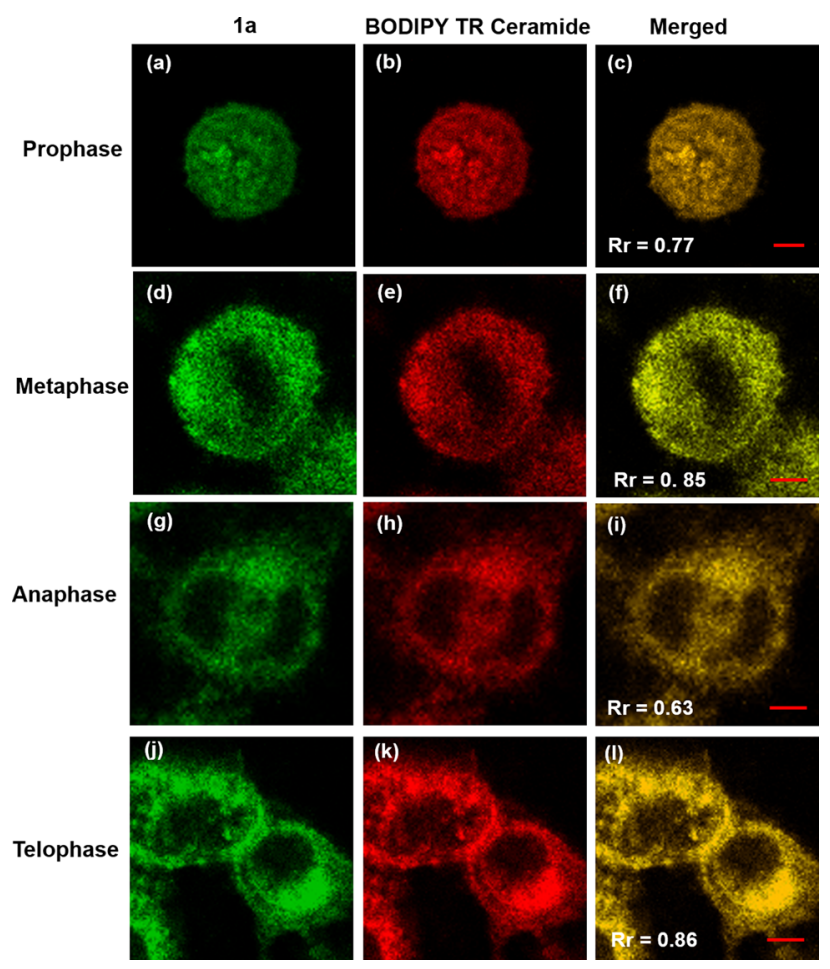

Figure 4. Subcellular localizations in synchronously dividing HeLa cells of 1a (a, d, g, j: $\lambda_{\text {ex }}=488 \mathrm{~nm}$; emission range $\left.489-580 \mathrm{~nm}\right)$ and BODIPY TR Ceramide (b, e, h, k: $\lambda_{\text {ex }}=633 \mathrm{~nm}$; emission range 634$740 \mathrm{~nm}$ ), and the merged images (c, f, i, 1). Scale bar: $5 \mu \mathrm{m}$.

Golgi imaging in vitro of $\mathbf{1 a}$ and $\mathbf{1 b}$ to the basicity of pyridyl moiety, considering that the Golgi apparatus is slightly acidic $(6.0-6.7) .^{37}$ Together, these colocalization results confirm that $\mathbf{l a}$ and $\mathbf{1 b}$ have the same intracellular localization as BODIPY TR Ceramide and can be potentially used as low cost Golgi probes to replace the more costly commercial ones.

Compared to one photon UV-excitation, two-photon microscopy (TPM), which utilizes a longer wavelength excitation source, owns the advantages of negligible background signal, deeper tissue penetration depth, reduced phototoxicity, and photobleaching. ${ }^{38}$ It is well-known that a strong ICT state is typically associated with two-photon absorption (TPA) characteristics, which can be beneficial for bioimaging applications. ${ }^{39,40}$ Given their remarkable ICT character, we also tested $\mathbf{1} \mathbf{a}$ and $\mathbf{1 b}$ for two-photon imaging capability in U2OS cells. The absorption maxima of the fluorophores are $\sim 400 \mathrm{~nm}$ in protic solvents, indicating that the optimal TPA wavelength is in the $780-800 \mathrm{~nm}$ range. To our delight, despite their small sizes, both of these two dyes exhibited very strong two-photon induced emission under the $790 \mathrm{~nm}$ laser excitation (Figure 5). The merged images also show perfect colocalizations in the Golgi apparatus with BODIPY TR Ceramide. The Rr values are 0.63 and 0.68 for $1 \mathrm{a}$ and $\mathbf{1 b}$, respectively. These initial results have demonstrated the imminent application potential of these ICTQs as both one- and two-photon fluorescence cell imaging.

In summary, we have conducted a highly selective and efficient condensation of $m$-phenylenebenzene with unsymmetric 1,3-diaketones to generate 2,4-disubstituted 7-aminoquinolines, by incorporating a strongly electron-withdrawing trifluoromethyl group. The introduction of such a trifluoromethyl group avoids the use of strong acid or other catalyst and also enhances the ICT process within 7-aminoquinolines 


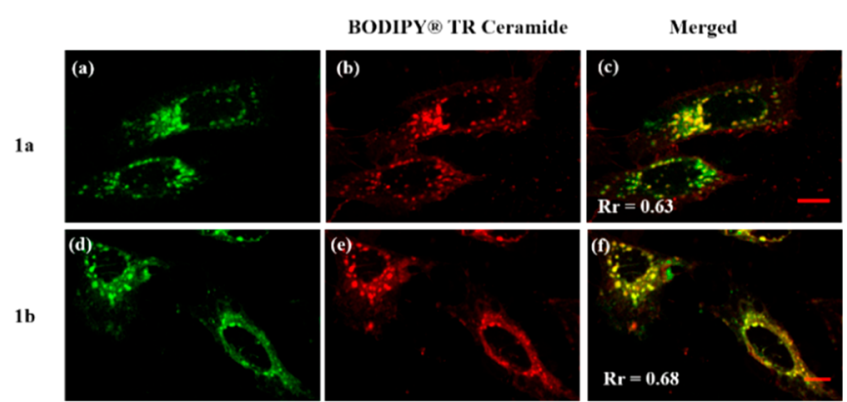

Figure 5. Two-photon fluorescence micrographs showing stained Golgi apparatuses within U2OS cells by $1 \mathbf{a}(\mathrm{a})$ and $\mathbf{1 b}(\mathrm{d})\left(\lambda_{\mathrm{ex}}=790\right.$ $\mathrm{nm}$; emission range $371-580 \mathrm{~nm}$ ); one-photon fluorescence micrographs showing stained Golgi apparatuses by BODIPY TR Ceramide $\left(\mathrm{b}, \mathrm{e}, \lambda_{\mathrm{ex}}=633 \mathrm{~nm}\right.$; emission range $\left.634-740 \mathrm{~nm}\right)$; merged images showing almost perfect overlaps between the quinoline and BODIPY TR Ceramide dyes (c, f). Scale bar: $10 \mu \mathrm{m}$.

from the strongly electron-donating amine group and the strongly electron-withdrawing trifluoromethyl group. These novel 7-aminoquinolines show large bathochromic shift going from nonpolar solvents to highly polar solvents. Interestingly, two of the new 7-aminoquinolines appear to exhibit high specificity for the Golgi apparatus as demonstrated by colocalization experiments with a commercial Golgi apparatus marker, BODIPY TR Ceramide, in various cell lines (HeLa, U2OS, and 4T1 cells). Furthermore, the dyes have exhibited potentials in both one- and two-photon fluorescence cell imaging. These preliminary findings provide new insights into developing low cost probes and drug delivery engineering with small quinoline molecules for Golgi apparatus. Currently, we are exploring the synthesis of red-emitting ICT quinolines for more useful life science applications.

\section{ASSOCIATED CONTENT}

\section{S Supporting Information}

The Supporting Information is available free of charge on the ACS Publications website at DOI: 10.1021/acsmedchemlett.9b00118.

Detailed experimental procedures and spectral data for all new compounds (PDF)

\section{AUTHOR INFORMATION}

\section{Corresponding Authors}

*E-mail: liguisheng@shnu.edu.cn.

*E-mail: xiaofengchen@shnu.edu.cn.

\section{ORCID $\odot$}

Jun Jiang: 0000-0002-6116-5605

Guoqing Zhang: 0000-0003-3155-8915

Guisheng Li: 0000-0002-4522-8936

Xiaofeng Chen: 0000-0002-5399-9781

\section{Author Contributions}

All authors have given approval to the final version of the manuscript.

\section{Funding}

This work was supported by the National Natural Science Foundation of China (21876113 to G. Li, 21803038 to X. Chen).

\section{Notes}

The authors declare no competing financial interest.

\section{ACKNOWLEDGMENTS}

We thank Dr. Rui Fu (RNA Bioscience Initiative, University of Colorado School of Medicine) for the helpful discussion about cell imaging.

\section{ABBREVIATIONS}

ICT, intramolecular charge transfer; $A D M Q$ 7-amino-2,4dimethylquinoline; ICTQs, intramolecular charge transfer quinolines; TPA, two-photon absorption; HOMO, highest occupied molecular orbital; LUMO, lowest unoccupied molecular orbital.

\section{REFERENCES}

(1) Adsule, S.; Barve, V.; Chen, D.; Ahmed, F.; Dou, Q. P.; Padhye, S.; Sarkar, F. H. Novel schiff base copper complexes of quinoline-2 carboxaldehyde as proteasome inhibitors in human prostate cancer cells. J. Med. Chem. 2006, 49, 7242-7246.

(2) Upadhyay, K. D.; Dodia, N. M.; Khunt, R. C.; Chaniara, R. S.; Shah, A. K. Synthesis and biological screening of pyrano[3,2c] quinolone analogues as anti-inflammatory and anticancer agents. ACS Med. Chem. Lett. 2018, 9, 283-288.

(3) Laras, Y.; Hugues, V.; Chandrasekaran, Y.; Blanchard-Desce, M.; Acher, F. C.; Pietrancosta, N. Synthesis of quinoline dicarboxylic esters as biocompatible fluorescent tags. J. Org. Chem. 2012, 77, 8294-8302.

(4) Wu, D.; Chen, L.; Ma, S.; Luo, H.; Cao, J.; Chen, R.; Duan, Z.; Mathey, F. Synthesis of 1,3-azaphospholes with pyrrolo[1,2-a]quinoline skeleton and their optical applications. Org. Lett. 2018, 20, 4103-4106.

(5) Foley, M.; Tilley, L. Quinoline antimalarials: mechanisms of action and resistance and prospects for new agents. Pharmacol. Ther. 1998, 79, 55-87.

(6) Greene, J. A.; Jones, D. S.; Podolsky, S. H. Therapeutic evolution and the challenge of rational medicine. N. Engl. J. Med. 2012, 367, $1077-1082$

(7) Kaur, K.; Jain, M.; Reddy, R. P.; Jain, R. Quinolines and structurally related heterocycles as antimalarials. Eur. J. Med. Chem. 2010, 45, 3245

(8) Singh, S. K.; Singh, S. A brief history of quinoline as antimalarial Agents. Int. J. Pharm. Sci. Rev. Res. 2014, 25, 295.

(9) Li, G.; Zhu, D.; Xue, L.; Jiang, H. Quinoline-based fluorescent probe for ratiometric detection of lysosomal pH. Org. Lett. 2013, 15, $5020-5023$

(10) Ahamed, B. N.; Ghosh, P. A chelation enhanced selective fluorescence sensing of $\mathrm{Hg}^{2+}$ by a simple quinoline substituted tripodal amide receptor. Dalton Trans 2011, 40, 12540-12547.

(11) Sutariya, P. G.; Pandya, A.; Lodha, A.; Menon, S. K. Fluorescence switch on-off-on receptor constructed of quinoline allied calix[4]arene for selective recognition of $\mathrm{Cu}^{2+}$ from blood serum and $\mathrm{F}^{-}$from industrial waste water. Analyst 2013, 138, 25312535.

(12) Mikata, Y.; Ugai, A.; Ohnishi, R.; Konno, H. Quantitative fluorescent detection of pyrophosphate with quinoline-ligated dinuclear zinc complexes. Inorg. Chem. 2013, 52, 10223-10225.

(13) Yang, W.; Yan, J.; Springsteen, G.; Deeter, S.; Wang, B. A novel type of fluorescent boronic acid that shows large fluorescence intensity changes upon binding with a carbohydrate in aqueous solution at physiological pH. Bioorg. Med. Chem. Lett. 2003, 13, 1019-1022.

(14) Skraup, V. Z. H.; Vorläufige, M. Eine Synthese des Chinolins. Bull. soc. Chira. 1880, 28, 62.

(15) Misani, F.; Bogert, M. T. The search for superior drugs for tropical diseases. II. Synthetic studies in the quinoline and phenanthroline series. Skraup and Conrad-Limpach-Knorr reactions. J. Org. Chem. 1945, 10, 347-365. 
(16) Gladiali, S.; Chelucci, G.; Mudadu, M. S.; Gastaut, M. A.; Thummel, R. P. Friedländer synthesis of chiral alkyl-substituted 1, 10phenanthrolines. J. Org. Chem. 2001, 66, 400-405.

(17) Eisch, J. J.; Dluzniewski, T. Mechanism of the Skraup and Doebner-von Miller quinoline syntheses: cyclization of, /3-unsaturated $\mathrm{N}$-aryliminium salts via 1,3 -diazetidinium ion intermediates. $J$. Org. Chem. 1989, 54, 1269.

(18) Buu-Hoi, N. P.; Royer, R.; Xuong, N. D.; Jacquignon, P. The Pfitzinger reaction in the synthesis of quinoline derivatives. J. Org. Chem. 1953, 18, 1209-1224.

(19) Combes, A. Quinoline synthesis. Bull. Chim. Soc. France 1888, 49, 89-94.

(20) Lodish, H.; Bark, A.; Zipersky, S. L.; Matsudaira, P.; Baltimore, D.; Darnell, J. Molecular Cell Biology, 4th ed; W. H. Freeman: New York, 2000.

(21) Aridor, M.; Hannan, L. A. Traffic jam: a compendium of human diseases that affect intracellular transport processes. Traffic 2000, 1, 836.

(22) Zhang, J. X.; Li, H.; Chan, C. F.; Lan, R.; Chan, W. L.; Law, G. L.; Wong, W. K.; Wong, K. L. A potential water-soluble ytterbiumbased porphyrin-cyclen dual bio-probe for Golgi apparatus imaging and photodynamic therapy. Chem. Commun. 2012, 48, 9646-9648.

(23) Zhang, H.; Fan, J.; Wang, J.; Zhang, S.; Dou, B.; Peng, X. An off-on COX-2-specific fluorescent probe: targeting the golgi apparatus of cancer cells. J. Am. Chem. Soc. 2013, 135, 11663-11669.

(24) Singh, H.; Lee, H. W.; Heo, C. H.; Byun, J. W.; Sarkar, A. R.; Kim, H. M. A Golgi-localized two-photon probe for imaging zinc ions. Chem. Commun. 2015, 51, 12099-12102.

(25) Haberhauer, G.; Gleiter, R.; Burkhart, C. Planarized intramolecular charge transfer: a concept for fluorophores with both large stokes shifts and high fluorescence quantum yields. Chem. - Eur. J. 2016, 22, 971-978.

(26) Suzuki, Y.; Yokoyama, K. Design and synthesis of intramolecular charge transfer-based fluorescent reagents for the highlysensitive detection of proteins. J. Am. Chem. Soc. 2005, 127, 1779917802.

(27) Maliakal, A.; Lem, G.; Turro, N. J.; Ravichandran, R.; Suhadolnik, J. C.; DeBellis, A. D.; Wood, M. G.; Lau, J. Twisted intramolecular charge transfer states in 2-arylbenzotriazoles: fluorescence deactivation via intramolecular electron transfer rather than proton transfer. J. Phys. Chem. A 2002, 106, 7680-7689.

(28) Shavaleev, N. M.; Scopelliti, R.; Graetzel, M.; Nazeeruddin, M. K.; Pertegas, A.; Roldan-Carmona, C.; Tordera, D.; Bolink, H. J. Pulsed-current versus constant-voltage light-emitting electrochemical cells with trifluoromethyl-substituted cationic iridium (iii) complexes. J. Mater. Chem. C 2013, 1, 2241-2248.

(29) Galievsky, V. A.; Zachariasse, K. A. Intramolecular charge transfer with N,N-Dialkyl-4-(trifluoromethyl)anilines and 4(dimethylamino)benzonitrile in polar solvents. Investigation of the excitation wavelength dependence of the reaction pathway. Acta Phys. Polym., A 2007, 112, S39-S56.

(30) Filler, R.; Kobayashi, Y.; Yagupolskii, L. M. Organofluorine Compounds in Medicinal Chemistry and Biomedical Applications; Elsevier, 1993.

(31) Bégué, J. P.; Bonnet-Delpon, D. Bioorganic and Medicinal Chemistry of Fluorine; John Wiley \& Sons, 2008.

(32) Ojima, I. Fluorine in Medicinal Chemistry and Chemical Biology; John Wiley \& Sons, 2009.

(33) Su, Q.; He, M.; Wu, Q.; Gao, W.; Xu, H.; Ye, L.; Mu, Y. The supramolecular assemblies of 7-amino-2, 4-dimethylquinolinium salts and the effect of a variety of anions on their luminescent properties. CrystEngComm 2012, 14, 7275-7286.

(34) Xu, S.; Evans, R. E.; Liu, T.; Zhang, G.; Demas, J.; Trindle, C. O.; Fraser, C. L. Inorg. Chem. 2013, 52, 3597-3610.

(35) Grabowski, Z. R.; Rotkiewicz, K.; Rettig, W. Aromatic difluoroboron $\beta$-diketonate complexes: effects of $\pi$-conjugation and media on optical properties. Chem. Rev. 2003, 103, 3899-4032.
(36) Zinchuk, V.; Grossenbacher-Zinchuk, O. Recent advances in quantitative colocalization analysis: focus on neuroscience. Prog. Histochem. Cytochem. 2009, 44, 125-172.

(37) Casey, J. R.; Grinstein, S.; Orlowski, J. Sensors and regulators of intracellular pH. Nat. Rev. Mol. Cell Biol. 2010, 11, 50-61.

(38) Helmchen, F.; Denk, W. Deep tissue two-photon microscopy. Nat. Methods 2005, 2, 932-940.

(39) Woo, H. Y.; Liu, B.; Kohler, B.; Korystov, D.; Mikhailovsky, A.; Bazan, G. C. Solvent effects on the two-photon absorption of distyrylbenzene chromophores. J. Am. Chem. Soc. 2005, 127, 1472114729.

(40) Wang, G.; Pu, K. Y.; Zhang, X.; Li, K.; Wang, L.; Cai, L.; Ding, D.; Lai, Y. H.; Liu, B. Star-shaped glycosylated conjugated oligomer for two-photon fluorescence imaging of live cells. Chem. Mater. 2011, 23, 4428-4434. 